\title{
The role of ambulatory hysteroscopy in reproduction
}

\author{
Lynne L L Robinson, ${ }^{1}$ Natalie A M Cooper, ${ }^{2}$ T Justin Clark ${ }^{3}$
}

\begin{abstract}
${ }^{1}$ Sub-Specialty Registrar in Reproductive Medicine, Birmingham Women's Hospital, Birmingham, UK

${ }^{2}$ Specialty Trainee in Obstetrics and Gynaecology, Birmingham Women's Hospital,

Birmingham, UK

${ }^{3}$ Consultant Obstetrician and

Gynaecologist, Birmingham Women's Hospital and Honorary Senior Lecturer, The University of Birmingham, Birmingham, UK
\end{abstract}

\section{Correspondence to}

Dr Lynne Robinson, Birmingham Women's Hospital, Mindelsohn Drive, Edgbaston, Birmingham B15 2TG, UK; lynne.robinson@blueyonder.co.uk

Received 3 July 2012 Revised 2 October 2012 Accepted 12 November 2012 Published Online First 24 January 2013
To cite: Robinson LLL, Cooper NAM, Clark TJ. Journal of Family Planning and Reproductive Health Care 2013:39:127-135.

\begin{abstract}
Hysteroscopy is a mainstay of modern gynaecologic practice. However, the role of ambulatory hysteroscopy and associated procedures has increased dramatically in recent years. The outpatient setting has associated benefits, both for the patient and economically. The advent of less invasive vaginoscopic techniques means that diagnostic hysteroscopy is achievable safely, comfortably and efficiently in almost all women and avoids the risk of a general anaesthetic.

This review aims to summarise first the role for ambulatory hysteroscopy in diagnosis of conditions contributing to reproductive failure. The second section of the review concentrates on the therapeutic interventions that can be performed hysteroscopically in the ambulatory setting such as tubal catheterisation, tubal occlusion and uteroplasty. Lastly, we discuss the role outpatient hysteroscopy plays in established contraceptive techniques such as intrauterine device placement, and the more recent advent of hysteroscopic sterilisation.
\end{abstract}

\section{INTRODUCTION}

Outpatient operative hysteroscopy is a relatively recent development, enabling treatment previously done in the operating theatre to be performed in the ambulatory setting without the need for general anaesthesia. A 'see and treat' style of management is being adopted, which is changing how we organise our services. Small diameter hysteroscopes are used with miniature mechanical or electrical instruments to treat patients in efficient one-stop clinics. The ambulatory setting in general is preferable for the patient and lowers costs, avoids complications and allows a quicker recovery time. ${ }^{1-3}$ There is, however, a lack of data regarding the cost effectiveness of testing strategies during routine infertility work up.

Abnormal bleeding disorders are the commonest reason for use of ambulatory hysteroscopy services. Diagnosis can be made by direct visualisation of intrauterine pathology or hysteroscopically guided endometrial biopsy. Efficient 'one-stop' diagnosis can be augmented by concomitant treatments of identified pathologies: the so-called 'see and treat' philosophy. Established interventions include treatment of focal uterine pathologies (polyps and small fibroids) as well as global ablative treatment of the endometrium. These treatment approaches will be discussed in a future review article in this Journal.

Reproductive issues are the most frequent indication for outpatient hysteroscopy (OPH) after bleeding disorders. Reproductive indications for referral include subfertility, recurrent or late miscarriage and fertility control. This review will address the role of $\mathrm{OPH}$ in the diagnosis of reproductive failure and contemporary approaches to treatment in an ambulatory setting. The utility of therapeutic hysteroscopy will be examined and in particular its place in enabling access to the Fallopian tubes to potentially enhance fertility via tubal catheterisation (TC), as well as eradication of fertility following hysteroscopic placement of tubal microinserts, namely hysteroscopic sterilisation.

\section{REPRODUCTIVE FAILURE}

\section{Diagnostic hysteroscopy}

Subfertility

Uterine factors can contribute to subfertility and recurrent implantation failure. Common pathologies include congenital uterine anomalies, or acquired lesions such as intracavity adhesions, fibroids and polyps. Whilst ultrasound scan is still the most common initial investigation for abnormal bleeding and fertility problems, hysteroscopy can be used in conjunction with this. Hysteroscopy is the 'gold standard' test for detecting focal pathologies, and incorporating endoscopic examination 
of the uterine cavity into the diagnostic work-up of subfertility allows identification and treatment of potentially correctable pathology, which is found in up to $45 \%$ of patients with recurrent implantation failure. ${ }^{4} 5$ Saline infusion sonography also has good diagnostic accuracy in detecting focal pathologies but does not have the therapeutic advantages of hysteroscopy.

Endometrial polyps are commonly found in women scheduled to have an in vitro fertilisation (IVF) procedure $^{467}$ and there is evidence that polypectomy prior to fertility treatment substantially improves the clinical pregnancy rate. ${ }^{49}$ The significance of polyps in subfertility and IVF may depend upon their type, size and location and so it remains unclear whether all polyps need removing. However, in the absence of complications, such as iatrogenic uterine synechiae, and the ability to simply remove polyps at the time of $\mathrm{OPH}$ using miniature mechanical and electrosurgical instruments (e.g. Gynecare Versapoint ${ }^{\mathrm{TM}}$, Ethicon, Somerville, NJ, USA) many gynaecologists would opt to remove them when found in association with subfertility work-up.

Delivering an electrosurgical treatment via minihysteroscopes avoids cervical dilatation and limits patient discomfort. In the outpatient setting, miniature bipolar electrodes (e.g. Gynecare Versapoint) are widely used because they can be used down standard small diameter operating hysteroscopes. Monopolar snares for removing polyps are available (e.g. Cooks Medical Europe, Limerick, Ireland) but do not have the safety advantages of bipolar systems (isotonic distension media, localised current) and so are less frequently employed.

Uterine fibroids are common in women of reproductive age, with an incidence of $20-40 \%,{ }^{10}$ the prevalence being higher in Afro-Caribbean women. A systematic review has shown that women with submucous fibroids have a reduced pregnancy rate and that hysteroscopic resection can improve pregnancy rates. ${ }^{11}$ As with polyps, submucous fibroids can be removed in an outpatient setting at the time of diagnostic hysteroscopy. This capability has been enhanced with the advent of technologies such as miniature hysteroscopic morcellators, which require a specific hysteroscope with an offset eyepiece (TRUCLEAR ${ }^{\circledR}$, Smith \& Nephew, Andover, MA, USA) and MyoSure ${ }^{\circledR}$ (Hologic, Marlborough, MA, USA). ${ }^{12}$ These devices use mechanical cutting to reduce the lesion into small chips and consequently evacuating these chips out of the uterine cavity by aspiration. Studies using this technique report short operating times and minimal complications for polyps and fibroids $<3 \mathrm{~cm}$, with rare conversion to the resectoscope. ${ }^{13}{ }^{14}$ In the outpatient setting, the use of the morcellator has also been reported as being successful and well tolerated for septum removal. ${ }^{15}$

However, the removal of most intracavity fibroids requires general anaesthetic so that larger diameter operative hysteroscopes, morcellators and resectoscopes can be employed. Even if a procedure under general anaesthetic is likely to be required, preliminary outpatient hysteroscopic assessment allows for optimal treatment planning, for example, evaluation of the most appropriate treatment setting, the need for endometrial preparation, type of instrumentation and likelihood of needing repeated surgeries (two- or three-stage resections).

In the absence of any uterine pathology there is evidence from a meta-analysis of a small number of trials that hysteroscopy may be beneficial prior to a subsequent IVF cycle in women where a previous cycle has been unsuccessful. This showed a significant increase in pregnancy rate in women who underwent an $\mathrm{OPH}$ prior to their IVF cycle [relative risk $(\mathrm{RR})=1.75,95 \%$ CI 1.51-2.03]. ${ }^{9}$ In addition, on subset analysis, women who were found to have a normal uterine cavity at $\mathrm{OPH}$ also showed a significant improvement in pregnancy rate compared with controls $(\mathrm{RR}=1.63,95 \% \mathrm{CI}$ $1.35-1.98)$. Why a hysteroscopy should confer such a benefit is unclear, and theories such as release of cytokines and growth factors in response to endometrial manipulation have been proposed. ${ }^{16}{ }^{17}$ To confirm the finding of improved outcome, a multi-centre randomised controlled study of pre-IVF OPH in women with recurrent IVF implantation failure, known as the TROPHY (TRial of OutPatient HysteroscopY) trial, is currently in progress in order to ascertain whether this potential benefit of OPH is valid. ${ }^{18}$ While we await the results of this trial, OPH remains useful where previous embryo transfers have been difficult; a situation known to be associated with a reduction in the pregnancy rate. ${ }^{19}{ }^{20}$ By employing a vaginoscopic technique, $\mathrm{OPH}$ can be useful to help identify the angle of the cervical canal, exclude any obstructive lesions, and plot a course into the uterine cavity for subsequent attempts at transcervical embryo transfer.

\section{Miscarriage}

The role of $\mathrm{OPH}$ in recurrent or late miscarriage is contentious. Recurrent miscarriage occurs in $1 \%$ of couples and is largely unexplained. However, some uterine causes may underlie some instances. First-trimester miscarriage may be linked to an underdeveloped, hypoplastic uterus or related to a distorted cavity arising from congenital or acquired uterine anomalies. Acquired anomalies such as fibroids and polyps are amenable to removal. Minor, filmy adhesions are easily divided in the outpatient setting, but more fibrous agglutination of the uterine cavity with significant cavity distortion is not suitable for ambulatory treatment. Congenital anomalies such as septate and bicornate uteri can be difficult to distinguish at hysteroscopy but an idea of cavity dimensions and degree of cavity separation can be ascertained. A septum is formed from fibroelastic tissue and often appears as a narrow, pale structure with a sharp apex, 
whereas the fundal projection arising in a bicornuate uterus is often covered by thicker endometrium such that it has a wider, pinker appearance. Definitive diagnosis requires magnetic resonance imaging (MRI) or laparoscopic imaging in addition. Uterine septae can be removed in an outpatient setting but complete, large volume septae will require general anaesthesia (see later).

There is no evidence to support the use of OPH in women with a history of late miscarriage or preterm delivery. However, the test may be reassuring to women and clinicians that no structural uterine anomaly is contributory to the reproductive problem. Furthermore, any deficiency of the intravaginal cervix, especially where there has been previous cervical surgery, including previous cerclage, can be assessed, informing the likely feasibility and suitability of potential cervical cerclage techniques. Cervical incompetence cannot be diagnosed with any degree of certainty as the cervix is a dynamic structure, responsive to the hormonal and physical environment within pregnancy. However, a patulous internal cervical os combined with difficulty distending the uterine cavity at $\mathrm{OPH}$ due to retrograde leakage of distension media through the cervical canal may help support such a diagnosis in the presence of a suggestive past obstetric history.

\section{Therapeutic hysteroscopy}

Tubal catheterisation

Tubal catheterisation (TC) is a technique used to treat a proximal Fallopian tube blockage (PTB) diagnosed following hysterosalpinogram (HSG). The proximal segment of the Fallopian tube has a thick muscular wall, is narrow and has a reduced population of ciliated cells. This makes this tubal segment particularly prone to obstruction, initially by material that can flow back from the uterus, and then in the luteal phase of the cycle by secretions produced locally. As PTB generally occurs in morphologically normal tubes, selective salpingography (SS) and TC can offer successful recanalisation of the tube. ${ }^{21}$

The tubal perfusion pressure (TPP) is a measurement of the resistance to dye flow through the Fallopian tube. There is evidence that reduced fertility is associated with high TPP. $^{22}{ }^{23}$ Increased conception rates have been demonstrated in those women with an initial high TPP which is reduced following TC. ${ }^{23}$ Nevertheless, women who have persistent high TPPs after TC have a poorer outcome and therefore should probably be directed towards IVF earlier rather than later.

Selective salpingography and tubal catheterisation

SS and TC are both used in ambulatory care for PTB and performed preferably during the follicular phase of the menstrual cycle. SS is performed in an outpatient setting in an X-ray department, as for an HSG. The SS catheter is forwarded through the cervical canal and advanced by tactile sensation to the tubal ostium. Its position is checked fluoroscopically and, if satisfactory, dye is injected. If the obstruction is overcome then the tubal contour is outlined with contrast. If the obstruction persists then a guidewire is threaded through the inner cannula and advanced towards the obstruction. Gentle pressure is applied to overcome it. The guidewire is then withdrawn and contrast medium injected through the SS catheter to confirm patency.

This procedure has been adapted for use under direct hysteroscopic vision thereby avoiding exposure to ionising radiation. The technique used for hysteroscopic TC is similar. A $5-5.5 \mathrm{~mm}$ continuous flow operative hysteroscope is used with recourse to local direct or paracervical anaesthesia if cervical dilatation is necessary. A vaginoscopic approach is preferable, as is a $30^{\circ}$ endoscope to facilitate access to the cornual regions of the uterine cavity and proximity to the tubal ostia. A fine catheter is passed down the 5-7 Fr working channel of the hysteroscope and guided towards the tubal ostium (Figure 1). The catheter is pushed gently under vision into the tubal ostium and methylene blue dye instilled via a syringe through the lumen of the catheter. If this does not overcome the obstruction (i.e. the catheter cannot be passed into the tubal ostium or retrograde spill of dye is noted despite forward instillation pressure) a guidewire is 'rail-roaded' through the lumen of the catheter (Figure 2). The guidewire is pushed gently into the cornual portion of the tube and the instillation of dye repeated.

Hysteroscopic tubal cannulation can also be done in theatre as a day case under laparoscopic guidance and a dye test performed at the end of the procedure to assess tubal patency. In the ambulatory hysteroscopy setting, confirmation that PTB has been overcome can be inferred by (i) ease of passage dye without retrograde spill; (ii) pre- and post-procedure transvaginal pelvic ultrasound to look for free fluid within the pelvis and (iii) hysterosalpingo-contrast sonography or

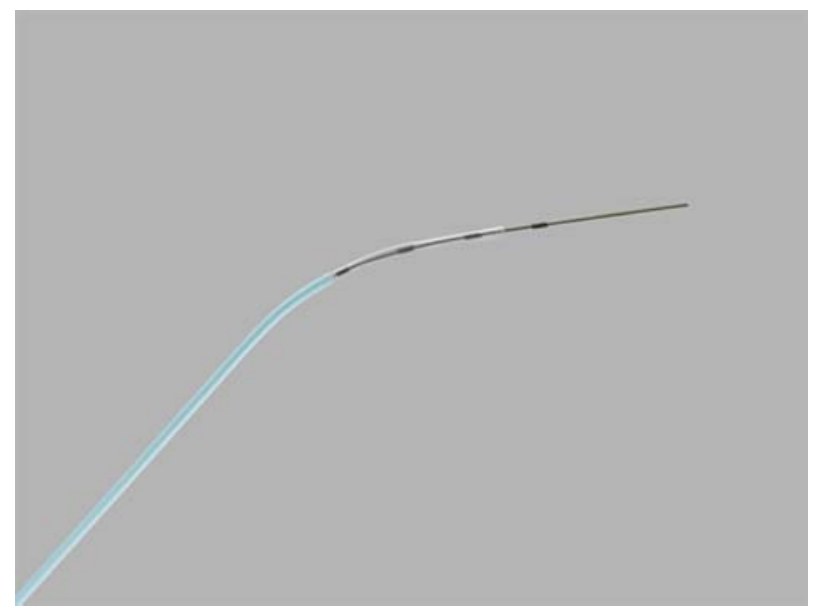

Figure 1 Catheter used for tubal catheterisation (Figure courtesy of Cooks Medical Europe, Limerick, Ireland). 


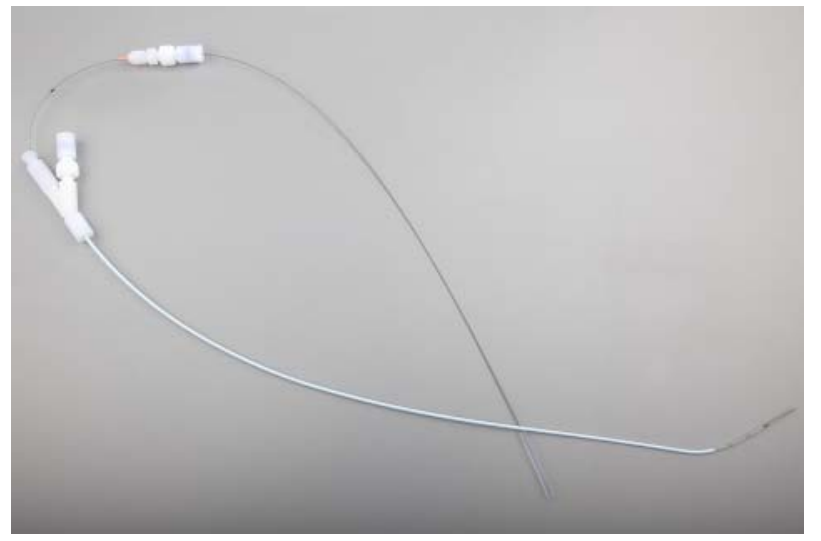

Figure 2 Catheter and guidewire used for tubal catheterisation (Figure courtesy of Cooks Medical Europe, Limerick, Ireland).

follow-up HSG arranged to confirm restoration of tubal patency.

\section{Success rates}

Data for hysteroscopic treatment of PTB are scarce, especially in the ambulatory setting. The procedure is well tolerated (Clark TJ, personal communication) using smaller diameter, continuous flow, operative hysteroscopy systems. However, visualisation within the uterus can be impaired once the tubal catheter is introduced where the working channel is integral to the inflow sheath. This results in loss of instillation pressure compromising cavity distension. This is more likely to be a problem with cases requiring difficult and repeated guidewire placements or in women with large uterine cavities or thickened, congested, secretory endometria. A careful, atraumatic technique ensuring good uterine distension prior to passage of the tubal catheter combined with periodic withdrawals of the catheter proximal to the inflow port to allow re-distension of the uterine cavity can help overcome this difficulty.

Most data available relate to SS rather than TC, although the studies of TC that are available report a recanalisation rate $25-87 \%$ and a pregnancy rate of $13-35 \% .{ }^{24-28}$ In the absence of extensive hysteroscopic data, data for the fluoroscopic approach of SS show a recanalisation rate of approximately $50 \%{ }^{23} 29$ although rates of up to $95 \%$ have been reported. ${ }^{2329} 30$ Between $20 \%$ and $40 \%$ of women become pregnant with follow-up of up to 2.5 years. $^{22} 23293031$ These are mainly spontaneous conceptions but some are achieved with ovulation induction or intrauterine insemination, both less invasive and considerably cheaper than IVF. It has been reported that the best chance for conception is in the first 3 months following TC. ${ }^{30}$ There is a hypothesis that PTB recurs in many women shortly after TC has been performed. ${ }^{30}$ However, follow-up HSGs after TCs confirm patency in $63-88 \%$ of women who have not conceived at 3-6 months. ${ }^{27}$ Also, a significant proportion (43\%) of conceptions occur after 12 months following treatment.

In many women PTB is unilateral. Therefore it is very difficult to demonstrate that recanalisation of the blocked Fallopian tube with TC does improve fertility for these patients. However, El Fekih et al. ${ }^{32}$ studied a series of 12 such women. Recanalisation of the PTB was successful in eight cases and four of them conceived spontaneously.

\section{Risks}

The procedure is well tolerated but some patients may suffer moderate pain and also a vasovagal reaction may occur. There is a small risk of pelvic infection and uterine trauma. Excessive forward pressure must be avoided, especially if using a fine guidewire, as this risks tubal perforation. This complication should be suspected where the patient experiences acute, sharp, localised pain as the serosal surface of the uterus is breached. The risk of tubal perforation during the procedure is approximately $2 \%$. If perforation is suspected then simple analgesics and broad-spectrum antibiotics should be given and the patient discharged after a short period of observation. The woman should be instructed to return if she experiences any ongoing pain, bleeding or fever. No other treatment is usually necessary as the perforation will seal closed spontaneously. In a small percentage of patients there may be intravasation of contrast agent. This can trigger an allergic reaction in those patients who are allergic to iodine, but the procedure would generally be avoided in these cases.

Occlusion of hydrosalpinges pre-IVF

The presence of hydrosalpinges in women embarking on an IVF procedure has been shown to significantly reduce the pregnancy and implantation rates. ${ }^{33}$ Occlusion of the tubes prior to IVF treatment shows a doubling of the clinical pregnancy rate. ${ }^{34-36}$ Hysteroscopic occlusion of the Fallopian tubes using the Essure ${ }^{\mathrm{TM}}$ system (Figure 3, see later for full details of this procedure) can be considered in women prior to IVF as treatment for hydrosalpinges. There may be some concern regarding the potential mechanical interference of the device on embryo transfer and implantation; however, there appears to be tissue encapsulation of the device after implantation. ${ }^{37}$ Several small studies have reported pregnancies from IVF following sterilisation with Essure. ${ }^{38-40}$ An important consideration is the potential effect of nickel ions within the uterine cavity on the developing fetus and therefore it is possible that pregnancy may be complicated by preterm delivery, miscarriage and fetal anomalies. However, in the pregnancies reported with Essure no fetal anomalies have been reported $^{38-40}$ and there was no cytotoxic, allergic or genotoxic activity in animal studies from the nickeltitanium alloy. ${ }^{41}$ Two cases have been reported to date of successful term pregnancies with IVF 


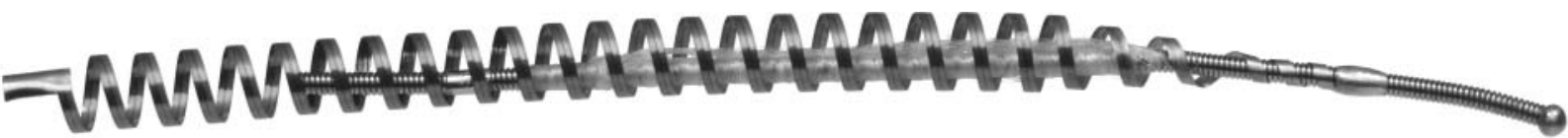

Figure 3 The Essure ${ }^{\mathrm{TM}}$ device (Figure courtesy of Conceptus Inc., Mountain View, CA, USA).

following Essure sterilisation. ${ }^{38}$ This is encouraging, and although there are genuine potential concerns regarding pregnancy post-Essure, in many women the benefits may outweigh the risks. Many women with multiple previous surgeries and tubes that are very inaccessible laparoscopically may be put at less risk by having their procedure done hysteroscopically.

Adhesiolysis and uteroplasty

Published data are lacking for plastic operations on the uterus in an outpatient, local anaesthetic setting. Hysteroscopic adhesiolysis can be achieved with scissors or Versapoint ${ }^{\circledR}$ diathermy in an outpatient setting where scar tissue is filmy and cavity distortion minimal. ${ }^{2}{ }^{42}$ General anaesthetic is required with ultrasonic or laparoscopic guidance where cavity distortion precluding easy anatomic delineation and/or fibrous adhesions is present. Similarly, uterine incisions longitudinally and/or horizontally with the aim of restoring the triangular uterine shape and increasing uterine capacity, in the presence of uterine hypoplasia, are best confined to general anaesthesia in a research setting. Uterine septae (complete or partial) are also effectively treated with miniature mechanical or electrosurgical instruments, but this fibro-elastic tissue is not devoid of sensory innervation and in the main hysteroscopic metroplasty is best done under general anaesthetic.

\section{CONTRACEPTION}

\section{Diagnostic hysteroscopy}

Prior to having an intrauterine device (IUD) fitted or tubal sterilisation, a diagnostic hysteroscopy in the outpatient setting is of great benefit. This can help treatment planning, and this role of $\mathrm{OPH}$ is often overlooked. For example, OPH may be useful in estimating the uterine size, capacity, shape and regularity and assessing the endometrium. The test can also help determine the most appropriate treatment setting (i.e. outpatient vs day case under general anaesthesia), based upon patient tolerance of the procedure and any adverse anatomical factors.

Abnormal uterine bleeding (AUB) is very common in women using hormonal contraception. OPH can assess the endometrium by direct visualisation and a directed biopsy can be taken if indicated. Polyps and fibroids are possible causes of AUB with or without contraceptives, and these can be visualised and treatment performed or subsequent treatment planned. Displacement of an IUD due to intracavitary leiomyomas are associated with unacceptable bleeding in women, especially those who have been bleed-free after prolonged use of the levonorgestrel-releasing intrauterine system (LNG-IUS). Ultrasonography and hysteroscopy may be useful in evaluating bleeding complaints in long-term users of this device. ${ }^{43}$

\section{Therapeutic hysteroscopy}

Hysteroscopic sterilisation

Female sterilisation is a common method of contraception throughout the world. The most widely available method of female sterilisation is the laparoscopic method using various techniques to block the Fallopian tubes such as Filshie clips, Falope rings, coagulation or cutting. Prior to the advent of laparoscopy, sterilisation was most commonly performed using the Pomeroy technique, which involves a mini-laparotomy. Both these techniques carry a risk of anaesthetic complications, pain, bowel injury, bleeding and infection.

Hysteroscopic sterilisation has been attempted using chemical and thermal methods of tubal damage and occlusion. However, whilst the transcervical route confers advantages over transperitoneal approaches, particularly avoidance of incisions, general anaesthesia and minimising inadvertent serious vascular or bowel injuries, comparable success rates in pregnancy prevention have not been achieved. This changed in 2001 with the introduction of the Essure method of hysteroscopic sterilisation (Conceptus, San Carlos, CA, USA). The procedure is performed vaginoscopically ${ }^{44}$ without the routine need for local anaesthesia or vaginal instrumentation. Pre-medication with nonsteroidal anti-inflammatory agents can help minimise peri and post-operative pain. ${ }^{45}$ Local cervical anaesthetic should be administered where cervical dilatation is required. The use of conscious sedation is favoured by some, but there is no evidence to support such an approach and risks inadvertent general anaesthesia. ${ }^{46}$

The Essure system consists of a Fallopian implant and a delivery catheter (Figure 3). The implant is a device $40 \mathrm{~mm}$ in length and $0.8 \mathrm{~mm}$ in diameter. The insert contains polyethylene fibres held in place by a stainless steel inner coil and a nickel-titanium alloy outer coil. The device is placed into the Fallopian tube via a standard, continuous flow hysteroscope $(\leq 5.5 \mathrm{~mm})$, using normal saline as the distension medium (Figure 4). Once the device is released hysteroscopically, the outer coil expands to approximately $2 \mathrm{~mm}$ to hold the insert in place in the Fallopian tube. The ideal placement is with between three and eight coils trailing within the uterus (Figure 5). More distal placement risks migration of the micro-inserts, whereas more proximal placement, especially where 


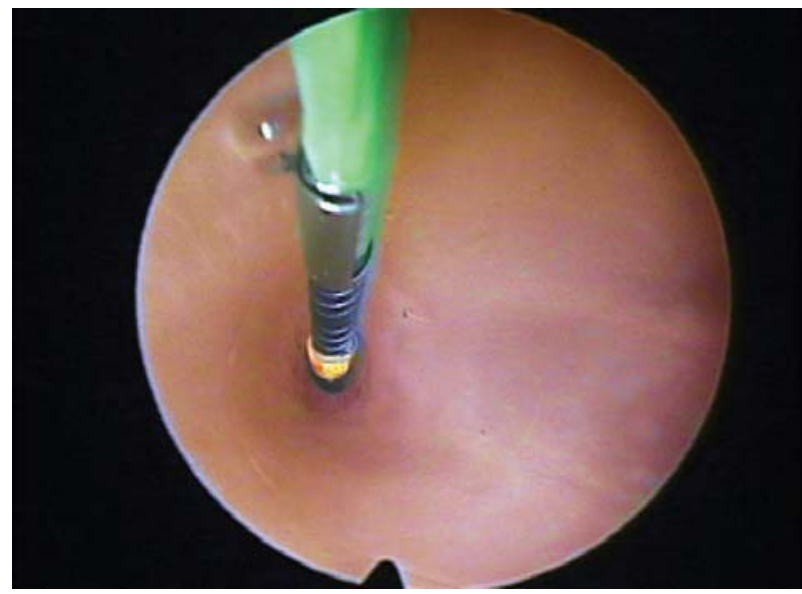

Figure 4 Essure ${ }^{\mathrm{TM}}$ insertion into tubal ostium (Figure courtesy of Conceptus Inc., Mountain View, CA, USA).

more than 20 trailing coils are visible, risks device expulsion.

Once in place, the device is designed to elicit a tissue reaction with growth of fibrous tissue around the insert causing proximal tubal blockage. The effect is not immediate and contraception is advised for at least 3 months after insertion to allow time for complete tubal occlusion to take place. ${ }^{47}$ Confirmatory radiology is scheduled at 3 months to confirm the adequacy of sterilisation; satisfactory device placement is checked by abdominal X-ray or transvaginal ultrasound $\operatorname{scan}^{48-50}$ in the $85 \%$ of procedures considered straightforward and the remainder undergo more invasive HSG to confirm tubal occlusion. ${ }^{51}$ As HSG is more expensive, painful and there is a risk of infection, allergic reaction and intravasation of dye, it is usually reserved for those women in whom the

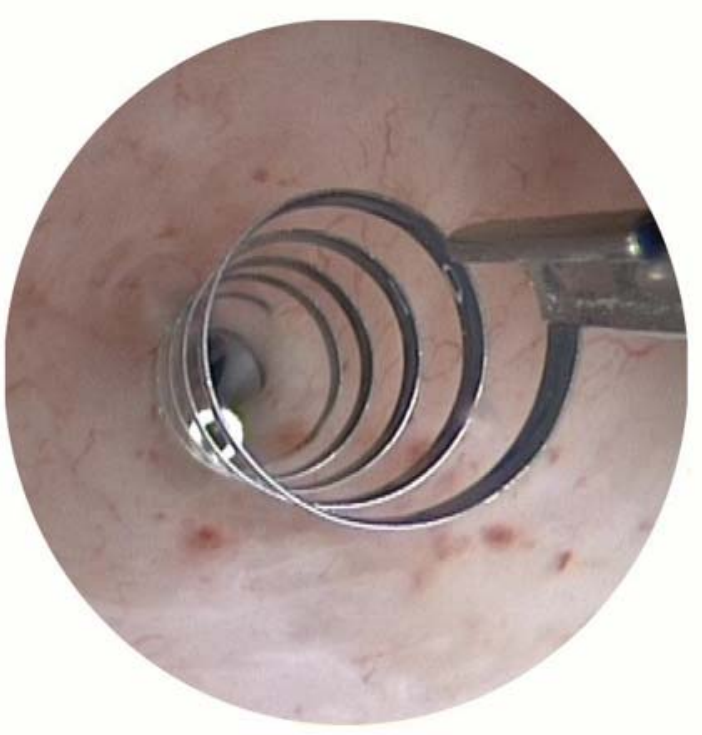

Figure 5 Essure ${ }^{\mathrm{TM}}$ device post-insertion into ostium (Figure courtesy of Conceptus Inc., Mountain View, CA, USA). transvaginal ultrasound scan is inconclusive. Transvaginal ultrasound with contrast infusion is an alternative. $^{52}$

\section{Benefits and risks}

In general, Essure is well tolerated and is quick, safe and provides effective contraception, certainly in the short term. Long-term follow-up is still lacking as the procedure is still relatively new. A systematic review on the subject was published in 2010, comprising two multicentre case series and five other studies, totalling 745 women. This reported bilateral placement rates of $81-98 \%$ (with up to two attempts). At 3 months, in $3.5 \%$ of women tubal occlusion could not be confirmed but at 6 months tubal blockage had been achieved in all women. ${ }^{53}$ The pivotal trial data ${ }^{54}$ from the original female cohort has recorded no pregnancies to date and the estimated cumulative 5-year effectiveness rate is $99.7 \% .{ }^{55}$ Based upon the number of reported cases of unintended pregnancy (mandatory in the USA) worldwide divided by the number of Essure kits sold worldwide corresponds to a failure rate of $0.15 \%(748 / 497305) .{ }^{56}$ Whilst this figure is likely to be an underestimation, the efficacy rate in general gynaecological practice, at least in the short and medium term, is consistent with published series and compares favourably with the leading laparoscopic technique in the UK. ${ }^{57}$ Whilst true method failures undoubtedly occur, non-compliance of women with the post-procedure protocol (i.e. abstinence from unprotected sex for at least 3 months, attendance for follow-up radiology) and misinterpretation of confirmatory radiology are the commonest reasons for failure. $^{565859}$

The procedure is safe and complications are usually minor and self-limiting, for example, vasovagal reactions, pain, cramping, nausea and bleeding. The risk of tubal perforation is $1-3 \%$ and intraperitoneally placed implants occur in $0.5-3 \%$ of cases. ${ }^{50} 60$ A recent retrospective study of more than 4000 patients reported a low complication rate of $2.7 \%$ and the majority of these were vasovagal reactions and no hospital admissions were required. ${ }^{61}$ It is probable that major complications will be reported with the more widespread adoption of hysteroscopic sterilisation and availability of longer-term follow-up data. However, the absence of any reported major perioperative injury is reassuring and demonstrates the safety advantages of an outpatient approach without anaesthesia and avoiding entry into the peritoneal cavity. As mentioned earlier there is a theoretical concern regarding the effects of nickel contained in the implant. However, in the pregnancies reported with Essure there was no cytotoxic, allergic or genotoxic activity in animal studies from the implant. ${ }^{41}$

Intrauterine contraceptive devices

IUDs are used by approximately 100 million women around the world today, making them the most 
popular form of reversible contraception, ${ }^{62}$ and almost $90 \%$ of users are in the developing world. The original IUDs were made of inert plastic, and these have gradually been replaced by copper-bearing or levonorgestrel-impregnated devices. Here we will consider the utility of $\mathrm{OPH}$ in relation to fitting, retrieving and managing complications of IUDs.

\section{Placement}

IUDs have long been successfully fitted in standard, community-based clinics. Where vaginal access may be limited or cervical dilatation is likely to be necessary, placement of devices can be more challenging. Thus in obese or nulliparous women, or those with a history of prior loop excision biopsies of the cervix or previous Caesarean sections, referral to an OPH unit should be considered. Standard operating couches in such clinics facilitate lithotomy positioning, thereby allowing better vaginal access and easier administration of local anaesthesia to the cervix. Patient exposure is greater, but this can be minimised with attention to the clinical environment, including provision of adjacent private changing facilities, use of patient gowns and sterile drapes. However, a gentler more expeditious approach is facilitated.

In addition to facilitating pelvic access and dilatation of the cervix, recourse to an $\mathrm{OPH}$ is useful where blind cervical dilatation is difficult. This situation usually arises in the presence of previous cervical or uterine surgery or where there is acute flexion, deviation or reduced mobility of the uterus, for example, following previous abdominal surgery, in the presence of uterine fibroids or pelvic endometriosis. Rather than persevere blindly with increased force (and patient discomfort), hysteroscopic visualisation is indicated. The axis of a deviated or tortuous cervical canal can be identified. Often a 'furrow' or overt false passage, where previous attempts at uterine instrumentation had run into trouble, can be seen. The hysteroscope can be advanced into the uterine cavity to exclude relevant uterine structural pathology and define the axis. Visualisation in this way enables confident redirection of dilatators and the IUD catheter and the preceding hydrodistension facilitates uterine instrumentation.

In addition to contraceptive requirements, the LNG-IUS is frequently employed for the alleviation of heavy menstrual bleeding symptoms and a concomitant outpatient diagnostic hysteroscopy can often be useful. This is particularly true where intrauterine pathology is likely, for example, failure of previous medical treatment, previous expulsion of IUDs or where transvaginal ultrasound has indicated focal lesions such as polyps or submucous fibroids. In the presence of focal pathology, the LNG-IUS is less effective and simultaneous treatment can be instigated (the so called 'see and treat', 'one-stop' philosophy) to optimise outcome.

\section{Retrieval}

The usual approach to retrieval and removal of 'lost' IUDs has been some combination of gentle probing of the endocervical canal with polyp or vascular forceps and/or instrumentation of the uterus with specifically designed plastic IUD retrieval devices. Failure results in a day case procedure under general anaesthesia (after a transvaginal ultrasound scan to confirm that the IUD is indeed in the uterus) to dilate the cervix and remove blindly with polyps forceps placed blindly within the uterine cavity. However, the latter procedure is anachronistic and unnecessary. Outpatient hysteroscopic removal, utilising small diameter continuous flow hysteroscopes with a 5-7 Fr grasping forceps placed down the working channel, is invariably successful, avoiding the need for analgesia, vaginal specula and inducing minimal patient discomfort. The likelihood of uterine trauma and infection is reduced by utilising direct vision because fruitless, repetitive attempts at blind location of IUDs are avoided. This is especially true where the threads of the IUD have eroded or been avulsed or where the IUD is clearly deformed, partially embedded or located within a uterine anomaly (e.g. the uterine horn of a bicornuate uterus, an adhesion or within an isthmocele; cystic defect in the anterior cervix at the level of the internal os/uterine isthmus arising from a Caesarean section scar).

\section{Complications}

In addition to hysteroscopic retrieval of 'lost' abnormally placed or partially embedded IUDs, OPH in conjunction with transvaginal ultrasound can be a useful diagnostic test for investigating and managing complications arising from the use of both hormonal and non-hormonal IUDs. Symptoms arising postplacement of IUDs, for example, persistent abnormal vaginal discharge, genital tract bleeding and pelvic pain, may indicate abnormal device placement or endometrial pathology within the uterine cavity. Dependent upon symptoms and signs, genital tract infection screening and/or antibiotic cover can be considered. Devices can be replaced with hysteroscopic assistance, focal pathologies such as polyps and small submucous fibroids removed and suspected endometrial disease biopsied.

\section{CONCLUSIONS}

The advent of $\mathrm{OPH}$ has allowed clinicians to investigate and treat patients in the ambulatory setting, without the need for general anaesthesia. This is true of women presenting with reproductive problems (i.e. fertility or contraceptive) as it is of women presenting with AUB. The miniaturisation of hysteroscopes and ancillary equipment, together with the adoption of less invasive vaginoscopic techniques, means that diagnostic hysteroscopy is achievable safely, comfortably and efficiently in almost all women. This is 
particularly important in women presenting with reproductive problems where traditional impediments to $\mathrm{OPH}$, namely uterine anomalies, nulliparity and possibly anxiety, are more prevalent.

Minor operative procedures of potential benefit to women with fertility problems can be undertaken simultaneously following diagnosis (e.g. polypectomy, myomectomy, minor adhesiolysis/uteroplasty, TC for PTB, and tubal occlusion for hydrosalpinges pre-IVF). This is especially beneficial in this population of women who have often already undergone multiple testing and interventions and who are keen to optimise and expedite assisted conceptive procedures. Recourse to general anaesthesia for either fitting or retrieving 'lost' IUDs is an anachronism. Even where placement of IUDs is considered potentially problematic (e.g. obesity, previous uterine/cervical surgery, nulliparity), concomitant OPH should be considered to aid outpatient fitting. This is undoubtedly preferable to routine admission for the procedure under general anaesthesia. The issue of consent with a 'see and treat' policy can be overcome by thorough counselling prior to the procedure and written information sent to the patient before their clinic attendance. Newer procedures such as hysteroscopic sterilisation provide a safer, more convenient choice of permanent fertility control, especially for those women with relative contraindications to laparoscopy.

Funding None.

Competing interests None.

Provenance and peer review Commissioned; externally peer reviewed.

\section{REFERENCES}

1 Marsh F, Kremer C, Duffy S. Delivering an effective outpatient service in gynaecology. A randomised controlled trial analysing the cost of outpatient versus daycase hysteroscopy. BJOG 2004;111:243-248.

2 Clark TJ, Gupta JK. Handbook of Outpatient Hysteroscopy. A Complete Guide to Diagnosis and Therapy (1st edn). London, UK: Hodder Education, 2005.

3 Saridogan E, Tilden D, Sykes D, et al. Cost-analysis comparison of outpatient see-and-treat hysteroscopy service with other hysteroscopy service models. J Minim Invasive Gynecol 2010;17:518-525.

4 Oliveira FG, Abdelmassih VG, Diamond MP, et al. Uterine cavity findings and hysteroscopic interventions in patients undergoing in vitro fertilization-embryo transfer who repeatedly cannot conceive. Fertil Steril 2003;80:1371-1375.

5 Makrakis E, Hassiakos D, Stathis D, et al. Hysteroscopy in women with implantation failures after in vitro fertilization: findings and effect on subsequent pregnancy rates. J Minim Invasive Gynecol 2009;16:181-187.

6 Fatemi HM, Kasius JC, Timmermans A, et al. Prevalence of unsuspected uterine cavity abnormalities diagnosed by office hysteroscopy prior to in vitro fertilization. Hum Reprod 2010;25:1959-1965.
7 Karayalcin R, Ozcan S, Moraloglu O, et al. Results of 2500 office-based diagnostic hysteroscopies before IVF. Reprod Biomed Online 2010;20:689-693.

8 Demirol A, Gurgan T. Effect of treatment of intrauterine pathologies with office hysteroscopy in patients with recurrent IVF failure. Reprod Biomed Online 2004;8:590-594.

9 El-Toukhy T, Sunkara SK, Coomarasamy A, et al. Outpatient hysteroscopy and subsequent IVF cycle outcome: a systematic review and meta-analysis. Reprod Biomed Online 2008;16:712-719.

10 Cramer SF, Patel A. The frequency of uterine leiomyomas. Am J Clin Pathol 1990;94:435-438.

11 Pritts EA, Parker WH, Olive DL. Fibroids and infertility: an updated systematic review of the evidence. Fertil Steril 2009;91:1215-1223.

12 Lukes AS. Myosure tissue removal system - comparative sedation study in an office setting. J Minim Invasive Gynecol 2011;176(Suppl. 1).

13 Hamerlynck TW, Dietz V, Schoot BC. Clinical implementation of the hysteroscopic morcellator for removal of intrauterine myomas and polyps. A retrospective descriptive study. Gynecol Surg 2011;8:193-196.

14 Emanuel $\mathrm{MH}$, Wamsteker K. The intra uterine morcellator: a new hysteroscopic operating technique to remove intrauterine polyps and myomas. J Minim Invasive Gynecol 2005;12:62-66.

15 Simons M, Hamerlynck TW, Abdulkadir L, et al. Hysteroscopic morcellator system can be used for removal of a uterine septum. Fertil Steril 2011;96:e118-e121.

16 Sharkey A. Cytokines and implantation. Rev Reprod 1998;3:52-61.

17 Basak S, Dubanchet S, Zourbas S, et al. Expression of pro-inflammatory cytokines in mouse blastocysts during implantation: modulation by steroid hormones. Am J Reprod Immunol 2002;47:2-11.

18 El-Toukhy T, Campo R, Sunkara SK, et al. A multi-centre randomised controlled study of pre-IVF outpatient hysteroscopy in women with recurrent IVF implantation failure: Trial of Outpatient Hysteroscopy - (TROPHY) in IVF. Reprod Health 2009;6:20.

19 Tomas C, Tikkinen K, Tuomivaara L, et al. The degree of difficulty of embryo transfer is an independent factor for predicting pregnancy. Hum Reprod 2002;17:2632-2635.

20 Sallam HN, Agameya AF, Rahman AF, et al. Impact of technical difficulties, choice of catheter, and the presence of blood on the success of embryo transfer - experience from a single provider. J Assist Reprod Genet 2003;20:135-142.

21 Allahbadia GN, Merchant R. Fallopian tube recanalization: lessons learnt and future challenges. Womens Health (Lond Engl) 2010;6:531-548, quiz.

22 Papaioannou S, Afnan M, Girling AJ, et al. The effect on pregnancy rates of tubal perfusion pressure reductions achieved by guide-wire tubal catheterization. Hum Reprod 2002;17:2174-2179.

23 Papaioannou S, Afnan M, Girling AJ, et al. The potential value of tubal perfusion pressures measured during selective salpingography in predicting fertility. Hum Reprod 2003;18:358-363.

24 Spiewankiewicz B, Stelmachow J. Hysteroscopic tubal catheterization in diagnosis and treatment of proximal oviductal obstruction. Clin Exp Obstet Gynecol 1995;22:23-27.

25 Darcy MD, McClennan BL, Picus D, et al. Transcervical salpingoplasty: current techniques and results. Urol Radiol 1991;13:74-79. 
26 Lang EK. Transvaginal recanalization of occluded fallopian tubes. Min Inv Ther 1995;4:129-135.

27 Kumpe DA, Zwerdlinger SC, Rothbarth LJ, et al. Proximal fallopian tube occlusion: diagnosis and treatment with transcervical fallopian tube catheterization. Radiology 1990;177:183-187.

28 Thurmond AS, Rosch J. Fallopian tubes: improved technique for catheterization. Radiology 1990;174:572-573.

29 Papaioannou S, Afnan M, Girling AJ, et al. Diagnostic and therapeutic value of selective salpingography and tubal catheterization in an unselected infertile population. Fertil Steril 2003;79:613-617.

30 Thurmond AS, Rosch J. Nonsurgical fallopian tube recanalization for treatment of infertility. Radiology 1990;174:371-374.

31 Verma A, Krarup K, Donuru A. Selective salpingography and fallopian tube catheterisation by guidewire. J Obstet Gynaecol 2009;29:315-317.

32 El Fekih C, Ouerdiane N, Mourali M, et al. Selective salpingography and tubal catheterization in infertile women. Tunis Med 2012;90:233-237.

33 Zeyneloglu HB, Arici A, Olive DL. Adverse effects of hydrosalpinx on pregnancy rates after in vitro fertilization-embryo transfer. Fertil Steril 1998;70:492-499.

34 Johnson NP, Mak W, Sowter MC. Laparoscopic salpingectomy for women with hydrosalpinges enhances the success of IVF: a Cochrane review. Hum Reprod 2002;17:543-548.

35 Johnson N, van VS, Sowter MC, et al. Surgical treatment for tubal disease in women due to undergo in vitro fertilisation. Cochrane Database Syst Rev 2010;(1):CD002125.

36 Johnson N, van VS, Sowter MC, et al. Tubal surgery before IVF. Hum Reprod Update 2011;17:3.

37 Kerin JF, Munday D, Ritossa M, et al. Tissue encapsulation of the proximal Essure micro-insert from the uterine cavity following hysteroscopic sterilization. J Minim Invasive Gynecol 2007;14:202-204.

38 Kerin JF, Cattanach S. Successful pregnancy outcome with the use of in vitro fertilization after Essure hysteroscopic sterilization. Fertil Steril 2007;87:1212-1214.

39 Mijatovic V, Veersema S, Emanuel MH, et al. Essure hysteroscopic tubal occlusion device for the treatment of hydrosalpinx prior to in vitro fertilization-embryo transfer in patients with a contraindication for laparoscopy. Fertil Steril 2010;93:1338-1342.

40 Galen DI, Khan N, Richter KS. Essure multicenter off-label treatment for hydrosalpinx before in vitro fertilization. J Minim Invasive Gynecol 2011;18:338-342.

41 Wever DJ, Veldhuizen AG, Sanders MM, et al. Cytotoxic, allergic and genotoxic activity of a nickel-titanium alloy. Biomaterials 1997;18:1115-1120.

42 Lindheim SR, Kavic S, Shulman SV, et al. Operative hysteroscopy in the office setting. J Am Assoc Gynecol Laparosc 2000;7:65-69.

43 Ronnerdag M, Odlind V. Late bleeding problems with the levonorgestrel-releasing intrauterine system: evaluation of the endometrial cavity. Contraception 2007;75:268-270.

44 Cooper NA, Smith P, Khan KS, et al. Vaginoscopic approach to outpatient hysteroscopy: a systematic review of the effect on pain. BJOG 2010;117:532-539.
45 Royal College of Obstetricians and Gynaecologists (RCOG). Best Practice in Outpatient Hysteroscopy (Green-top Guideline No. 59). London, UK: RCOG, 2011.

46 Mino M, Arjona JE, Cordon J, et al. Success rate and patient satisfaction with the Essure sterilisation in an outpatient setting: a prospective study of 857 women. BJOG 2007;114:763-766.

47 Valle RF, Carignan CS, Wright TC. Tissue response to the STOP microcoil transcervical permanent contraceptive device: results from a prehysterectomy study. Fertil Steril 2001;76:974-980.

48 Kerin JF, Levy BS. Ultrasound: an effective method for localization of the echogenic Essure sterilization micro-insert: correlation with radiologic evaluations. J Minim Invasive Gynecol 2005;12:50-54.

49 Weston G, Bowditch J. Office ultrasound should be the first-line investigation for confirmation of correct ESSURE placement. Aust N Z J Obstet Gynaecol 2005;45:312-315.

50 Thiel JA, Suchet IB, Lortie K. Confirmation of Essure microinsert tubal coil placement with conventional and volume-contrast imaging three-dimensional ultrasound. Fertil Steril 2005;84:504-508.

51 National Institute for Health and Clinical Excellence. Hysteroscopic Sterilisation by Tubal Cannulation and Placement of Intrafallopian Implants (IPG315). 2009. http://www.nice.org.uk/guidance/IPG315/Guidance/pdf [accessed 4 June 2012].

52 Connor V. Contrast infusion sonography in the post-Essure setting. J Minim Invasive Gynecol 2008;15:56-61.

53 Kerin JF, Cooper JM, Price T, et al. Hysteroscopic sterilization using a micro-insert device: results of a multicentre phase II study. Hum Reprod 2003;18:1223-1230.

54 Sinha D, Kalathy V, Gupta JK, et al. The feasibility, success and patient satisfaction associated with outpatient hysteroscopic sterilisation. BJOG 2007;114:676-683.

55 Bradley LD. Long-term follow up of hysteroscopic sterilization with the Essure micro-insert. Supplement to JMIG 2008;15:S15.

56 Veersema S, Vleugels MP, Moolenaar LM, et al. Unintended pregnancies after Essure sterilization in the Netherlands. Fertil Steril 2010;93:35-38.

57 Peterson HB, Xia Z, Hughes JM, et al. The risk of pregnancy after tubal sterilization: findings from the U.S. collaborative review of sterilization. Am J Obstet Gynecol 1996;174:1161-1168.

58 Levy B, Levie MD, Childers ME. A summary of reported pregnancies after hysteroscopic sterilization. J Minim Invasive Gynecol 2007;14:271-274.

59 Hurskainen R, Hovi SL, Gissler M, et al. Hysteroscopic tubal sterilization: a systematic review of the Essure system. Fertil Steril 2010;94:16-19.

60 Shavell VI, Abdallah ME, Diamond MP, et al. Placement of a permanent birth control device at a university medical center. J Reprod Med 2009;54:218-222.

61 Povedano B, Arjona JE, Velasco E, et al. Complications of hysteroscopic Essure ${ }^{\circledR}$ sterilisation: report on 4306 procedures performed in a single centre. BJOG 2012;119:795-799.

62 World Health Organization. Intrauterine Devices. Technical and Managerial Guidelines for Services. 1997. http://whqlibdoc.who. int/publications/1997/924154497X_(part1).pdf [accessed 4 June 2012]. 